\title{
Análisis de los criterios de selección en el acceso a estudios oficiales de Danza Analysis of selection criteria in the access tests to official Dance studies
}

\author{
Verónica Parent Mathias*, Jerónimo García Romero*, María José Campoy Sanchez**, José Ramón Alvero Cruz* \\ *Universidad de Málaga (España), **Conservatorio Profesional de Danza de Granada (España)
}

Resumen. El acceso a los estudios oficiales de danza se realiza mediante pruebas de aptitud en los Conservatorios Profesionales de Danza y son realizadas por profesores de danza que evalúan aptitudes rítmicas, expresivas y psicomotrices y por otra parte, por médicos que realizan una evaluación de características físicas. La selección de los alumnos se realiza en función de los resultados de ambas pruebas. El objetivo es conocer si existen diferencias entre el profesorado a la hora de calificar en función de su especialidad en danza y conocer las características principales que rigen sus calificaciones. No han sido encontrados estudios acerca de pruebas específicas para realizar dicha evaluación. Para cumplimentar los objetivos del estudio datos cuantitativos fueron recogidos con la aplicación de un cuestionario ad hoc $(\mathrm{n}=27)$. Los resultados muestran diferencias estadísticamente significativas en los cuales el profesorado de danza clásica priorizó calificaciones en el ítem peso/talla, morfología del pie y extensión de piernas, mientras los docentes de danza española y flamenco concedieron mayor importancia a la evaluación de la morfología de las piernas y aspectos de ritmo y expresión. Los profesores de la especialidad de contemporáneo destacaron las calificaciones de morfología de la columna y la pelvis.

Palabras clave. Evaluación, Test, Selección, Aptitud Física, Aptitud Artística, Danza.

Abstract. Access to official studies in dance is granted following dance aptitude tests in Spanish Professional Conservatories of Dance, performed by dance teachers who evaluate rhythmic, expressive and psychomotor skills and doctors physicians who perform an assessment of the physical characteristics. The selection of students is based on the results of these tests. The aim is to know if there are differences among teachers who evaluate according to their specialty in dance (Ballet, Flamenco, Contemporary or Spanish dance) and the main features determining their ratings. No studies have been found on tests to conduct such evaluations. To complete the objectives of the study, quantitative and qualitative data were collected through the administration of a test $(n=27)$. The results indicate that, while the classical dance teacher prioritized weight/height, foot morphology and leg flexibility, teachers of Spanish dance and flamenco granted greater importance to the evaluation of the morphology of the legs and aspects of rhythm and expression. Teachers of contemporary dance emphasized ratings of spine and pelvis morphology.

Key words. Assessment, Tests, Selection, Physical Aptitude, Artistic Ability, Dance.

\section{Introducción}

Los estudios de danza se realizan en escuelas públicas o privadas que dependen del Ministerio de Educación y Ciencia. En Andalucía existen Conservatorios Profesionales de Danza en Almería, Granada, Córdoba, Sevilla, Cádiz y Málaga. También existen algunas escuelas privadas que previamente homologadas por la Consejería de Educación de la Junta de Andalucía, imparten determinados cursos y ofrecen al alumno una titulación final según el curso realizado.

El acceso a los estudios oficiales de danza se realizan mediante Pruebas de Aptitud en los Conservatorios Profesionales de Danza de España, regulados en el Boletín Oficial de la Junta de Andalucía(BOJA, 2009a, 2009b, 2009c, 2009d) estas pruebas son realizadas por profesores de danza que evalúan aptitudes rítmicas, expresivas y psicomotrices y por médicos que realizan una evaluación de las características físicas de los aspirantes. Los aspirantes a los estudios de danza deben realizar dicha prueba de aptitud para conseguir los diferentes grados de capacitación y profesionalización que nos brinda la danza, dentro de unas características de estilo de vida saludable para el bailarín(Dick, 2006).

Las pruebas de aptitud constan de dos valoraciones, una estática y una específica. La estática es realizada por personal médico y se mide el peso y la talla y se inspecciona columna, pies, pelvis y piernas, sin especificar que se debe observar en dicha evaluación, otorgándose a esta una calificación máxima de 2 puntos. La valoración específica es realizada por el profesorado de danza del centro al cual se pretende acceder y consta de una valoración dinámica, una rítmica, psicomotriz y finalmente expresiva. Posee una calificación máxima de 8 puntos. En la tabla 1 se establecen los distintos descriptores publicados por la administración. Esta prueba de aptitud se puede realizar a partir de los 8 años cumplidos en el curso escolar al cual se pretende acceder aunque también la pueden realizar aspirantes con más edad.

La danza es una actividad física bastante exigente desde el punto de vista atlético, por lo que se echa de menos una valoración de las cualidades físicas básicas (Twitchett, Koutedakis, \& Wyon, 2009; Wilson \& Batson, 2014; Wilson \& Deckert, 2009) en los aspirantes a los estudios de danza. De hecho, la valoración de la condición física se ha estudiado

Fecha recepción: 28-02-15- Fecha envío revisores: 03-03-15- Fecha de aceptación: 25-08-15 Fecha recepción: $28-02-15-$ veronicaparent@hotmail.com ampliamente desde la Educación Física, estableciendo pruebas de aptitud física para su evaluación (Fetz \& Kornexl, 1976; Garth Fisher, 1996; Lopez Gutierrez, Linares Girela, \& Ruiz Rodriguez, 2010). Numerosos autores han estudiado las mismas, desde el ámbito de la Educación Primaria y Secundaria en nuestro país (Gras Garcia, 1985; Martinez, 2003; Martínez López, Zagalaz Sánchez, \& Linares Girela, 2003). En otra serie de disciplinas deportivas las pruebas de aptitud física son guiadas de forma específica según las cualidades físicas que ese deporte quiera evaluar. En el caso de la danza, además se suma a la valoración de cualidades, como la flexibilidad (F. Ayala, Sainz de Baranda, Cejudo, \& Santonja, 2012, 2013; Bozic, 2010) la más estudiada, la coordinación, la agilidad y fuerza y la evaluación de cualidades artísticas como la capacidad expresiva, la musicalidad y el ritmo (BOJA, 2009d). Sin embargo, no en todos los casos su utilización es adecuada ni su resultado garantiza una correcta evaluación, pudiendo tener influencia la edad del alumno evaluado (Martinez, 2003; Sicilia et al., 2006).

Las pruebas de aptitud física no son utilizadas homogéneamente por los profesores evaluadores. De forma general se afirma que aunque no está del todo claro que las pruebas de aptitud física ejerzan una función motivadora y evaluadora que beneficie el aprendizaje del alumnado, desde el punto de vista de la educación física si se considera que produce un efecto de superación. A través de la propia evaluación continua se puede presentar el deseo de alcanzar rendimientos cada vez mas altos y de superación de uno mismo y/o a los demás (Martinez, 2003). Por tanto surge la pregunta de si esto sería aplicable a las pruebas de aptitud física para una evaluación puntual de selección, que permitan el acceso a determinada enseñanza, y si realmente los criterios utilizados en las pruebas de selección son capaces de evaluar las capacidades de los aspirantes.

Los estudios sobre la concepción de la evaluación del profesorado no son muy numerosos hasta la fecha, siendo especialmente notable esta carencia en el profesorado de los Conservatorios de Música y Danza (Bautista Arellano, 2011). En la bibliografía consultada no se han encontrado estudios en la danza sobre las pruebas de aptitud y/o acceso o un estudio pormenorizado de los ítems que influyen en estas.

Otro aspecto a tener en cuenta sería el nivel educativo en el que el profesorado de danza ejerce su acción. La danza discurre en el marco de las Enseñanzas de Régimen Especial con un amplio rango de edad de alumnado, que suele comprender entre los ocho y los dieciocho años (siendo el margen superior muy amplio). Algunos resultados indican 
que mientras el profesorado de educación secundaria prioriza la evaluación de aprendizajes procedimentales, los docentes del nivel de educación primaria, conceden mayor importancia a la evaluación de aprendizajes actitudinales (Sicilia, et al., 2006). En nuestro caso además nos interesa la calificación de la prueba de aptitud. En la educación primaria y secundaria la calificación es mayoritariamente considerada como elemento necesario en el proceso de evaluación, si bien el profesorado de secundaria mostró estar más a favor de las calificaciones. (Sicilia, et al., 2006)

Por todo ello el objetivo principal del estudio es el análisis de los criterios de calificación de las pruebas de aptitud/selección para el ingreso en un Conservatorio Profesional de Danza en función de la formación del evaluador. Como objetivos secundarios nos planteamos obtener información del profesorado sobre los ítems establecidos en la Prueba de Aptitud y comprobar la correspondencia entre las calificaciones del profesorado y los baremos de la administración.

\section{Metodología}

\section{Participantes}

Los participantes fueron 27 profesores que realizan habitualmente pruebas de aptitud en los Conservatorios Profesionales de Danza de la Comunidad Autónoma de Andalucía o aquel profesorado que debidamente formado imparte clases para la preparación de las pruebas de aptitud como es el caso de las Escuelas Municipales de Danza, todos ellos con la misma titulación académica (Titulo Profesional de Danza).

El profesorado fue seleccionado en función de tres criterios de inclusión: estar activo en cualquiera de las cuatro especialidades de las Enseñanzas Profesionales de Danza (danza clásica, danza española, danza contemporánea o flamenco) en los cursos 2012-2013 y 20132014, ya que el periodo en el cual se enviaron los formularios a rellenar fue el año 2013, una experiencia mínima de enseñanza de cinco años y en esos cursos académicos haber preparado la prueba o haber formado parte de los tribunales de las pruebas de aptitud de los Conservatorios Profesionales de Danza que participaron en el estudio.

\section{Instrumentos}

El análisis de las calificaciones de la prueba de aptitud se obtuvieron a partir de las publicadas en página web oficial del Conservatorio Profesional a estudio.

Para obtener la información y realizar el estudio de la valoración de los ítems establecidos en las pruebas de aptitud se aplicó un cuestionario ad hoc que consta de dos partes que corresponden a dos páginas. En la primera se explicaba el proceso de cumplimentación y en la segunda se exponían los ítems de la prueba de aptitud que son calificados en la misma (tabla 1). Se divide en dos apartados:

1. Valoración médica (Estática): peso/talla, piernas, pelvis, pies y columna. Calificación total máxima 2 puntos.

2. Valoración por parte del profesorado de danza (Específica): flexión columna, extensión piernas, salto, ritmo, espacio-tiempo, lateralidad, memoria y expresión. Calificación total máxima 8 puntos.

Se solicitaba que se calificara por apartados según la importancia que el profesorado de danza daba a cada uno de los ítems en la prueba de aptitud, sin tener en cuenta el rango establecido por la Administración (Ver tabla 4). Por ejemplo, si consideraban que la valoración de la flexión de columna debía tener más importancia que la extensión de piernas así podían calificarla con más puntos, respetando siempre los rangos máximos.

Con este cuestionario se pretendía obtener la información de cual sería el rango de calificaciones que el profesor de danza encuestado daría a cada uno de los ítems establecidos por la administración para conocer a cuales de dichos ítems les da mayor importancia.

Se impuso el margen para la valoración médica de un máximo de dos puntos y para la valoración del profesorado en suma ocho puntos, obteniendo finalmente una calificación global de 10 puntos.

\section{Fiabilidad de los cuestionarios}

La fiabilidad test-retest entre cuestionarios fue evaluada en 15 profesores mediante la administración del mismo cuestionario a la semana, para comprobar la concordancia de las respuestas y siendo evaluado mediante el coeficiente de correlación intraclase(CCI).

\section{Procedimiento de obtención de datos}

Se solicitó la colaboración de los profesores a título individual. Se les explicó que se estaba realizando un estudio sobre «evaluación y calificación de las pruebas de aptitud» y les informamos del tipo de participación requerida. Una vez accedían a su participación, firmaron un consentimiento informado. Los cuestionarios fueron enviados por correo electrónico y devueltos por la misma vía en un plazo máximo de dos meses durante 2013, de ahí que puedan ser participantes en activo de dos cursos escolares (2012-2013 y 2013-2014). Se enviaron 35 cuestionarios obteniendo 27 respuesta válidas.

En los cuestionarios solo se indicaba la especialidad a la cual pertenecía el profesor (danza contemporánea, danza española, flamenco y danza clásica). Estos cuestionarios fueron remitidos a una tercera persona de forma anónima para realizar el estudio estadístico, solo detallando del participante su especialidad en danza.

Los grupos que se establecieron para el análisis fueron:

1. Profesorado de Danza Contemporánea (CO), $n=5$

2. Profesorado de Danza Española (ES), $n=8$

3. Profesorado de Flamenco (FL), $\mathrm{n}=5$

4. Profesorado de Danza Clásica (CL), $n=9$

Se analizaron los ítems en función de lo dispuesto en el BOJA (BOJA, 2009a, 2009d) siguiendo la misma clasificación que se facilita en el cuestionario.

\section{Análisis estadístico}

Los análisis estadísticos se realizaron con el programa MedCalc versión 13.2.0.0 (Mariakerke, Belgium). Se calculó la media de las medias de los valores dados y ésta es equivalente a la media aritmética calculada mediante la fórmula $\mathrm{Sx} £ \mathrm{~N}$. Los valores han sido expresados como media $\pm \mathrm{DE}$ (desviación estándar).

Un análisis mediante el test de Shapiro-Wilk demostró una distribución no normal. Se realizó un análisis estadístico no paramétrico para comparación de las medias mediante el test de Kruskall-Wallis. Si este mostraba al factor significativo, se procedía a una comparación no paramétrica post hoc entre grupos según Conover (Conover, 1999).

El estudio de fiabilidad del cuestionario se evaluó mediante el coeficiente de correlación intraclase, determinando una alta fiabilidad valores $>0.90$ (Shrout \& Fleiss, 1979). El nivel de significación aceptado fue establecido para un valor de $p<.05$.

\section{Resultados}

En primer lugar se expresan los resultados de las calificaciones de la prueba de aptitud de un curso académico de un Conservatorio Profesional de danza y posteriormente los resultados del cuestionario enviado al profesorado.

\begin{tabular}{|c|c|c|c|c|}
\hline Valoración estática & $\begin{array}{c}\begin{array}{c}\text { Valoración } \\
\text { dinámica }\end{array} \\
\end{array}$ & $\begin{array}{l}\text { Valoración } \\
\text { rítmica }\end{array}$ & $\begin{array}{l}\text { Valoración } \\
\text { psicomotriz }\end{array}$ & $\begin{array}{c}\text { Valoración } \\
\text { artística }\end{array}$ \\
\hline Peso y medida & $\begin{array}{l}\text { Flexión de } \\
\text { columna }\end{array}$ & $\begin{array}{l}\text { Capacidad } \\
\text { rítmica }\end{array}$ & Lateralidad & $\begin{array}{l}\text { Capadidad } \\
\text { expresiva y } \\
\text { deativa }\end{array}$ \\
\hline $\begin{array}{l}\text { Aptitudes físicas: columna, } \\
\text { pie, pelvis y piernas }\end{array}$ & $\begin{array}{l}\text { Extensión } \\
\text { depiernas } \\
\text { Salto }\end{array}$ & $\begin{array}{c}\text { Relación } \\
\text { espacio-tiempo }\end{array}$ & Memoria motriz & \\
\hline \multicolumn{5}{|c|}{$\begin{array}{l}\text { Tabla } 2 . \\
\text { Resultados as pirantes Pruebas de Aptitud. }\end{array}$} \\
\hline \multicolumn{3}{|l|}{ Aspirantes } & seleccionados & lista de espera \\
\hline \multicolumn{3}{|l|}{ Número } & 88 & 19 \\
\hline \multicolumn{3}{|l|}{ Nota media } & 7.7 & 7.2 \\
\hline \multirow{2}{*}{\multicolumn{3}{|c|}{ Con máxima puntuación en ambos apartados (n) }} & 2 & 1 \\
\hline \multirow{2}{*}{\multicolumn{3}{|c|}{$\begin{array}{l}\text { Con un } 2 \text { en el R. Médico y menos de } 8 \text { en la P. Aptitud } \\
\text { Con } 8 \text { en la P. Aptitud y menos de } 2 \text { en el R. Médico }\end{array}$}} & $2.2 \%$ & $5.2 \%$ \\
\hline & & & $\begin{array}{l}59 \% \\
11.3 \%\end{array}$ & ${ }_{5.2 \%}^{88.4 \%}$ \\
\hline \multicolumn{3}{|c|}{ Con menos de 2 en R. Médico y más de 5 en P.Apti tud } & $21.5 \%$ & $5.2 \%$ \\
\hline
\end{tabular}




\section{A. Calificaciones en las Pruebas de Aptitud}

De los 113 que solicitaron realizar las pruebas de aptitud, se presentaron 107. De estos fueron seleccionados 88, quedando pues, en lista de espera 19 aspirantes. En la tabla 2 se resume la descripción de los datos obtenidos de dichas calificaciones.

Se puede observar que la nota media de los aspirantes fue de 7.2 mientras que de los seleccionados fue de 7.7, quedándose en lista de espera un alumno con puntuación máxima. El 88.4\% de los aspirantes en lista de espera, tienen la máxima nota en la valoración estática, pero tienen menor calificación en la valoración específica. El porcentaje de aspirantes con máxima puntuación en ambos apartados es superior en los aspirantes que no fueron aceptados, un $5.2 \%$ frente a un $2.2 \%$ de los que consiguieron superar la prueba de aptitud. Un aspirante puede puntuar muy alto en la parte expresiva de la valoración específica y no tanto en las pruebas de evaluación física de la parte estática, y de esta manera compensar unas puntuaciones con otras e ingresar en los Estudios Oficiales de Danza.

\section{B. Resultados del estudio de evaluación y calificación de la Prueba de Aptitud}

Se recogieron 27 formularios completos, pertenecientes a cinco centros diferentes, que representa una participación del $77 \%$, ya que el número total de formularios enviados fue de 35.

En la tabla 3 se detallan las medias obtenidas de los valores que cada profesor ha considerado indicado para cada ítem, teniendo en cuenta el rango de 0-2 puntos para la valoración estática y de 0-8 para la valoración dinámica, distribuyendo los ítems no de forma proporcional, sino de la forma que ellos consideraran mas importante para el acceso de los aspirantes. en la tabla 3. A continuación destacamos, según cada ítem, la calificación media de todas las especialidades (tabla 4) y si existen diferencias estadísticamente significativas entre los grupos en la valoración estática o en la valoración dinámica.

\section{Valoración estática}

Enla valoración del ítem peso/talla se obtuvo una calificación media 0.51 puntos, siendo en CL donde se observó una mayor puntuación, encontrando diferencias significativas, con los otros grupos estudiados $(\mathrm{CO}, \mathrm{ES}$ y FL) $(p<.05)$.

En la columna se obtuvo un valor medio de 0.40 puntos, siendo en CO donde se obtuvo mayor puntuación. Se observan diferencias estadísticamente significativas $(p<.05)$ de nuevo entre el grupo CL con respecto a los otros, siendo en este caso la puntuación media menor que en el resto.

De nuevo se observan diferencias estadísticamente significativas $(p<.05)$ del ítem pie del grupo de CL con respecto a los otros tres grupos, con una media de 0.31 puntos.

Con respecto a la pelvis existen diferencias estadísticamente significativas $(p<.05)$ entre CO y los grupos de ES y CL, teniendo CO en este caso la menor de las puntuaciones medias con respecto a los otros grupos como se puede observar en la tabla 4 , con una media de 0.38 puntos.

En el ítem piernas se obtuvo una media de 0.42 puntos. Se encuentran diferencias estadísticamente significativas $(p<.05)$ entre el grupo de FL con respecto a ES y CL.

Valoración dinámica

Para la flexión de la columna se obtuvo una media 0.91 puntos,

Tabla 3.
Medias y desvi ación están dar de los items según la especial idad de danza del profesorado evaluado



A continuación se describen los resultados según cada ítem desarrollado en el cuestionario. Éstos son: peso/talla, columna, pie, pelvis, piernas, flexión columna, extensión piernas, salto, ritmo, espacio-tiempo, lateralidad, memoria y expresión. Los cuatro primeros ítems son los referidos a la parte de valoración estática (realizada por un médico) y los otros son los que conforman la parte de valoración dinámica (dirigida y valorada por profesionales y profesores de danza).

Las calificaciones obtenidas del profesorado estudiado se observan

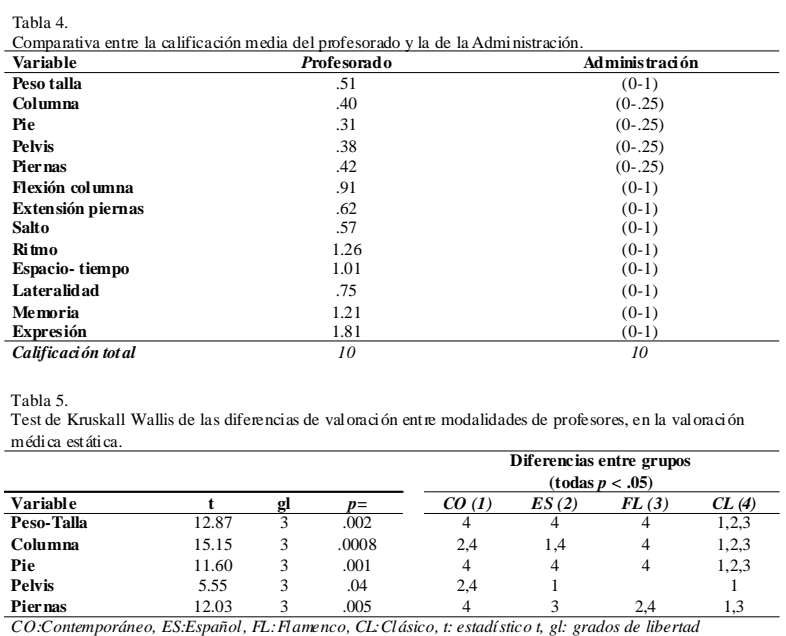

otorgando el CL la mayor valoración con 1 punto de medio, siendo estadísticamente significativo $(p<.05)$ con respecto a FL

En la extensión de piernas se obtuvo una media de 0.62 puntos, siendo CL quien le otorga una puntuación mayor con una significación estadística $(p<.05)$ con respecto a ES.

Para el ítem del salto se obtuvo una media de 0.57 puntos, sin hallarse diferencias significativas $(p>.05)$ entre las diferentes especialidades.

En el caso del ritmo se calcula una media de las medias de 1.26 puntos, encontrando en las especialidades de FL y CL, la mayor puntuación, hallándose diferencias estadísticamente significativas $(p<.05)$ con respecto a CO y ES.

Con respecto al ítem de espacio/tiempo se obtuvo una media de 1.01 puntos. Es la especialidad de ES quien otorga una mayor puntuación, siendo estadísticamente significativa $(p<.05)$ con respecto a las otras 3 especialidades (CO, FL y CL)

La lateralidad obtuvo una media de 0.75 puntos, siendo como en el ítem anterior la especialidad de ES quien otorga una mayor puntuación, estadísticamente significativa $(p<.05)$ con respecto a CO, FL y CL.

Para la memoria se obtuvo una media de las medias de 1.21 puntos. Observamos que la mayor puntuación es para la especialidad de flamenco con diferencias significativas $(p<.05)$ respecto a CO, ES y CL.

En la expresión, se obtuvo una media de 1.81 puntos, siendo las puntuaciones mayores las otorgadas por $\mathrm{CO}$ y FL, con diferencias estadísticamente significativas $(p<.05)$ con respecto a los grupos CL y ES. 


\begin{tabular}{|c|c|c|c|c|c|c|c|}
\hline \multirow{2}{*}{ Variable } & \multirow[b]{2}{*}{$t$} & \multirow[b]{2}{*}{ gl } & \multirow[b]{2}{*}{$p=$} & \multicolumn{4}{|c|}{$\begin{array}{l}\text { Diferencias entre grupos } \\
\text { (todas } p<.05 \text { ) }\end{array}$} \\
\hline & & & & $C O(1)$ & $E S(2)$ & $F L(3)$ & $C L(4)$ \\
\hline Flexión columna & 6.61 & 3 & .02 & & & 4 & 3 \\
\hline Extensión piemas & 8.82 & 3 & .01 & & 4 & & 2 \\
\hline Salto & 2.43 & 3 & .42 & & & & \\
\hline Ritmo & 14.88 & 3 & .0008 & $2,3,4$ & $1,3,4$ & 1,2 & 1,2 \\
\hline Espado-Tiempo & 12.74 & 3 & .0001 & 2 & $1,3,4$ & 2 & 2 \\
\hline Later alidad & 13.81 & 3 & .0009 & 2 & $1,3,4$ & 2 & 2 \\
\hline Memoria & 11.34 & 3 & .004 & 3,4 & 3 & $1,2,4$ & 1,3 \\
\hline Expresión & 18.11 & 3 & .0002 & 2,4 & 1,3 & 2,4 & 1,3 \\
\hline
\end{tabular}

\section{Fiabilidad del cuestionario}

La fiabilidad del cuestionario (test-retest) fue establecida mediante el coeficiente de correlación intraclase, que fue de 0.987 (95\% IC : 0.961 $-0.99)$

\section{Discusión}

Por los resultados encontrados en el presente estudio se ha comprobado que los profesores de las diferentes especialidades de danza, califican de forma diferente los distintos ítems de la prueba de aptitud. Ya en el BOJA se sugiere: «Para las pruebas de aptitud el tribunal será único y estará compuesto por un profesor o profesora de danza clásica y un profesor o profesora de danza española, designados por la Dirección del centro, y un médico o médica que será designado por la persona titular de la correspondiente Delegación Provincial de la Consejería competente en materia de educación. Para las pruebas de acceso a cursos distintos del de primero, existirá un tribunal formado por tres profesores o profesoras designados por la Dirección del centro» (BOJA, 2009d, p.11).

Vistos los resultados de la prueba de aptitud de en un conservatorio, resaltamos que los aspectos que entendemos cruciales, son la edad de los aspirantes y el número de plazas ofertadas en el momento de la Prueba de Aptitud. La edad viene marcada por la legislación, siendo los ocho años la edad propuesta como idónea para el inicio de los estudios oficiales (BOJA, 2009a, 2009c, 2009d). Alumnos con una puntuación muy alta pueden quedar excluidos por no tener ocho años. Si los aspirantes tienen mayor edad, pueden realizar pruebas a otros cursos (las denominadas Pruebas deAcceso), pero la preparación para dichas pruebas requiere conocimientos prácticos y técnicos, ya que se evalúan los conocimientos de danza hasta el curso a examen, y éstos deberán adquirirse fuera del ámbito oficial y gratuito del Estado en academias privadas. Las plazas ofertadas por los Conservatorios son limitadas.

La valoración estática realizada por médicos debería establecer homogeneidad en los criterios de evaluación, con rangos de valoración de cara a una valoración más fácil y reproducible. Estos rangos de calificación deben de estar en consonancia con los aspectos más importantes de la condición morfológica del bailarín a evaluar para realizar una buena selección de alumnos en la danza.

La morfología y las condiciones del aparato locomotor se pueden definir en cada momento y en función de los resultados, calificarlas. Las características morfológicas de los bailarines o las valoraciones de movilidad articular pueden variar de una especialidad a otra. Las características morfológicas de un bailarín difieren con respecto a los de la población en general (Ferrari, Silva, Martins, Fidelix, \& Petroski, 2013) y en algunos casos entre especialidades de danza como la danza deportiva o bailes de salón (Liiv, Wyon, \& Jürimae, 2014). Las bailarinas de contemporáneo tienen una mayor mesomorfia que las de ballet clásico, siendo estas últimas las que suelen tener menor IMC y menor porcentaje de grasa corporal (Liiv, et al., 2013, 2014). Entre especialidades de la danza como la clásica, la española y el flamenco no se han encontrado diferencias en el porcentaje graso valorado tanto por métodos antropométricos como por bioimpedancia eléctrica (Alvero-Cruz, Marfell-Jones \& Alacid, 2014).

En el peso y la talla, una de las cuestiones a debatir es la manera de clasificar a los aspirantes. La edad idónea para realizar la prueba de aptitud son los ocho años (cumplir dicha edad en el curso escolar al cual aspira matricularse) y por lo tanto la valoración de la misma no debería realizarse simplemente como tal, sólo tomando el peso y la talla, sino estableciendo rangos entre tablas de percentiles y/o tablas de índice de masa corporal (IMC). Estos resultados no deben tener rangos cerrados ya que los IMC varían según la edad y el crecimiento (Cole, Bellizzi, Flegal, \& Dietz, 2000). Así pues, creemos que las calificaciones deberían de estar adaptadas al contexto de la danza y la edad del aspirante, ya que existen diferencias entre las especialidades, como la danza clásica con menor proporción grasa y menor IMC o el contemporáneo con mayor masa muscular (Liiv et al., 2013, 2014; Marra et al., 2009; Mihajloviæ \& Mijatov, 2003). Esto, en alguna medida podría estar influido por la importancia que en CL se le da al fenotipo, a las figuras estilizadas y probablemente siempre asociado a un relativo bajo peso, para poder conseguir objetivos (Ferrari et al., 2013). No se debe olvidar que este ítem no viene estratificado por lo cual un IMC bajo, no se especifica como debe de ser evaluado. Existen niños con altos IMC debido a que tienen un mayor desarrollo de la masa libre de grasa, que otros y además esta variable nos puede informar acerca de trastornos como el retraso de crecimiento o problemas de conducta alimentaria (Clarkson, Freedson, Skrinar, Keller, \& Carney, 1989; Hergenroeder, Fiorotto, \& Klish, 1991; Yannakoulia et al., 2004; Yannakoulia, Keramopoulos, Tsakalakos, \& Matalas, 2000). Estudios de la composición corporal basados en la evaluación del componente de grasa y/o de la masa muscular, nos podría dar información para poder evaluar a los aspirantes y realizar su seguimiento en los diferentes cursos. Se deberían realizar estudios más amplios para determinar la validez longitudinal de los estudios de composición corporal o de la morfología sobre las calificaciones y sus cambios (Husted, Cook, Farewell, \& Gladman, 2000).

Con respecto al ítem columna, debemos tener en cuenta que el rango de movimiento de la espalda en danza clásica es mayor, aunque evidentemente en las bailarinas de flamenco también esta aumentado con respecto a la población general (Gomez-Lozano \& Santonja Medina, 2012). En nuestro estudio el grupo CL obtuvo una puntuación menor, con respecto a las otras especialidades. Ésto puede ser debido a que la puntuación de la primera parte del cuestionario debe ser repartida entre estos cinco ítems y si le damos mayor importancia al peso/talla en el CL, existe por tanto, una menor puntuación en los otros apartados. La columna debe ser evaluada para discriminar si existen alteraciones morfológicas que dificulten los estudios en danza, recomendándose el cribado y la identificación de los bailarines escolióticos, pues parece existir una relación entre la escoliosis y la dorsalgia (Steinberg, et al., 2013).

Es conocida la importancia del pie en la disciplina de CL, pues no poseer una buena flexión plantar comporta no conseguir una correcta postura sobre las puntas y por ello dificultades técnicas importantes y provocarunmayoríndice de lesiones de sobrecarga (Pearson \& Whitaker, 2012). En contraposición, la disciplina de FL, donde el pie juega un papel fundamental en ritmo y fuerza, pero no tanto en necesidad de flexión plantar, obtuvo menos puntuación en la valoración del profesorado quizás porque son las características de movilidad en flexión y extensión las que se evalúan en las pruebas de aptitud. Las características de los pies son difíciles de evaluar, sobretodo teniendo en cuenta la edad del aspirante. Debemos evaluar patologías importantes o malformaciones como el hallux valgus o los pies cavos que dificultarían la practica deportiva, o podrían variar la biomecánica del movimiento del pie, como en el zapateado, (Echegoyen, Aoyama, \& Rodríguez, 2013; J. Russell, 2008) o en el caso de las puntas (Ritter, 2008), por tanto puede provocar lesiones (J. A. Russell, Kruse, Koutedakis, McEwan, \& Wyon, 2010). Las alteraciones morfológicas pueden causar disfunciones en las cadenas cinéticas y ser causa de lesiones en pie y tobillo de bailarines (Macintyre, 2000).

En relación a la valoración de la pelvis y la movilidad de la cadera y en concreto la posibilidad de realizar una abducción en rotación (el conocido «en dehors») no suele ser tal imprescindible para el desarrollo del CO, aunque evidentemente un buen «en dehors» siempre enriquecerá los movimientos de la danza. Sin embargo es el «en-dehors», es la actividad propia de la danza más evaluada, siendo este un criterio de 
selección y evaluación (Francisco Ayala, Sainz de Baranda, De Ste Croix, \& Santonja, 2012; Drê_ewska, Ga³uszka, \& Sliwiñski, 2012; Gilbert, Gross, \& Klug, 1998; Khan et al., 2000). el flamenco, la morfología de las piernas son fundamentales para su ejecución, y cualquier alteración previa en las mismas (genu varo, genu valgo, etc.) podría provocar cambios en las cadenas cinéticas y por consecuencia lesiones importantes en el bailarín, por ello probablemente sea el profesorado de FL el que mas valor de al ítem «piernas».

El zapato de tacón se ha relacionado con el desarrollo de patologías músculo-esqueléticas, en diversas localizaciones como articulación metatarsofalángica del primer dedo (Wang, Hsu, Shau, Shieh, \& Hsu, 1999), tendón de Aquiles (Carlson, Fleming, \& Hutton, 2000), rodilla, sobre todo articulación femoropatelar (Kerrigan, Todd, \& Riley, 1998) y en columna lumbar (Bejjani et al., 1988; Pozo Municio, 2003). El profesorado de CO en nuestro estudio le da más importancia a ítems, como las piernas o la columna en los primeros años de formación. Las variaciones estáticas patológicas, como el «genu recurvatum», se pueden relacionar con variaciones dinámicas en la ejecución de la danza y conllevar dolor repetido en pies, tobillos, rodillas, siendo factores condicionantes el stress tendinoso-muscular en ciertas regiones provocado por dichas patologías (Blanco \& Ortigosa, 1989).

El objetivo en las Enseñanzas Básicas, es dar a conocer y promover la danza en sus diferentes estilos y por ello, la valoración estática de la prueba de aptitud debería de ir encaminada a evaluar diferentes patologías especificas que puedan dificultar la realización de la danza, siendo de carácter más diagnóstico y de reconocimiento médico (F. Ayala et al., 2012; García, Medina, \& Pastor, 1996; Gomez-Lozano \& Santonja Medina, 2012; Medina, Ortuño, \& Moro, 1994; Sainz de Baranda \& Santonja, 2009; Santonja Medina, 2001) como se realiza en otras actividades atléticas (Coplan, 2002). Las desalineaciones del aparato locomotor que se observan con mas frecuencia durante la etapa escolar o la pubertad, no se agravan por la práctica de la danza de iniciación, por lo cual, no está contraindicada, pero deberían, bajo nuestro punto de vista, quedar registradas ya que al aumentar la carga de entrenamiento con los siguientes años de formación, podrían llegar a transformarse en patologías estructuradas (Santonja Medina, 2001). Por ejemplo, en caso de diagnosticar alguna variación de la normalidad (genu valgo de $6 \mathrm{~cm}$ ) debería quedar registrado y calificarlo en función de la edad y gracias a esta evaluación inicial poder realizar un seguimiento. Esto permitiría una valoración más amplia y global, por ejemplo en la prevención de acortamientos y descompensaciones musculares (Ramos Espada, Gonzalez Montesinos, \& Mora Vicente, 2007).

La valoración dinámica de la prueba de aptitud, tiene en cuenta los aspectos expresivos, rítmicos y de musicalidad, aunque estos pueden ser más difíciles de evaluar y calificar ya que parten de la observación y opinión más subjetiva. Por otra parte, también de debe alcanzar una competencia cultural y artística (Montaivez, 2012), por ejemplo mediante ejercicios de expresión corporal (Amada, González, \& Montávez, 2013). En nuestro caso esta competencia artística se valora independientemente, como el caso del grupo FL que le otorga más importancia a la memoria, la expresión y el ritmo.

Por todo esto en la prueba de aptitud pequeñas variaciones en las calificaciones pueden hacer que un aspirante se quede fuera de los estudios. No existen unos criterios que delimiten rangos de normalidad, como ocurre con el ítem peso/talla, en el que la Administración nos indica un rango de calificación de 0 a 1 punto, pero no establece una norma o valores de talla y/o peso, a que corresponda la máxima o mínima calificación. Dentro de cada criterio/ítem se deberían conocer cuales son los apartados a observar y medir y establecer unos mínimos para la misma. Por ejemplo, si se valora dentro del ítem «pelvis», la movilidad articular de la cadera, se debe establecer el rango de «en dehors», calificándolo según grados de apertura

La calificación global de una prueba de aptitud, tan importante como para permitir el acceso a unos estudios oficiales en el sistema educativo, se hace con una calificación numérica pero sin establecer los rangos de normalidad y/o de excelencia para poder calificar. De esta forma, la nota es computada según el criterio del tribunal calificador y como se ha comprobado existen diferencias entre las especialidades de danza. Además las pruebas deben ser reproducibles para realizar una evaluación continua. (Bozic, 2010; Brushøj, Langberg, Larsen, Nielsen, \& Hölmich, 2007; Pozo Municio, 2003).

\section{Propuesta de rango de calificaciones}

En base a los resultados obtenidos, se propone un rango de calificación mas adecuado a las medias que han sido presentadas (ver tabla 7). En posteriores estudios se deberían estudiar cuales son las valoraciones que se deben hacer en cada uno de ellos, para poder estratificar las calificaciones como tales. La formación del profesorado de danza y del médico, así como la inclusión en los tribunales de las nuevas especialidades de los Conservatorios como danza Contemporánea y el Flamenco puede disminuir estos sesgos. Se propondría así mismo la creación de un documento de consenso que establezca los nuevos rangos de calificación, más acordes con el tratamiento equitativo, atención a la diversidad y las propias evaluaciones del profesorado de danza y del personal examinador. Sería un buen comienzo para dar equidad a estas pruebas, además de servir como evaluación continua de los alumnos seleccionados.

\begin{tabular}{lc} 
Tabla 7. Prop uesta de calific ación. & \\
\hline Variable & Propuesta de rango \\
\hline Peso talla & $(0-.5)$ \\
Columna & $(0-4)$ \\
Pie & $(0-.3)$ \\
Pelvis & $(0-.4)$ \\
Piernas & $(0-0.4)$ \\
Flexión columna & $(0-1)$ \\
Extensión piernas & $(0-.6)$ \\
Salto & $(0-6)$ \\
Ritmo & $(0-1)$ \\
Espacio- tiempo & $(0-1)$ \\
Lateralidad & $(0-8)$ \\
Memoria & $(0-1)$ \\
Expresión & $(0-2)$ \\
\hline Calificación total & 10
\end{tabular}

\section{Limitaciones del estudio}

Para ulteriores estudios podríamos aumentar el número de participantes, realizándolo de forma multicéntrica y extensible a otras Comunidades Autónomas, para poder realizar una comparativa intercomunidades.

\section{Conclusiones}

Las pruebas de aptitud son un método de selección y la puerta de entrada al sistema educativo de Régimen Especial en danza. Existen diferencias entre los cuatro grupos de profesorado (danza clásica, danza española, flamenco y contemporáneo) en la evaluación de las pruebas de aptitud, por lo cual el tribunal debería de estar formado por especialistas de las diferentes áreas presentes en los Conservatorios de Danza.

Los resultados indican que el profesorado de danza clásica prioriza las calificaciones en el ítem peso/talla, en la morfología del pie y extensión de piernas, mientras que los docentes de danza española y flamenco conceden mayor importancia a la evaluación de la morfología de las piernas y aspectos de ritmo y expresión. Los profesores de la especialidad de contemporáneo destacan las calificaciones de morfología de la columna y la pelvis.

Para evitar la variabilidad entre los evaluadores se debería buscar un tribunal mixto en el cual estén representados profesionales de diferentes disciplinas para evitar sesgos derivados de aspectos relacionados con la práctica profesional de cada evaluador. La valoración estática realizada por médicos debería estar en consonancia con los aspectos más importantes de las condiciones del bailarín a evaluar.

Queda patente la importancia de la edad de comienzo, que muestra un sesgo en la elección y mayor facilidad para entrar si el aspirante tiene ocho años.

En vista a los resultados el ítem «peso y talla» debería ser calificado en función de la edad de los aspirantes y como dato para evaluación continua, no para evaluación selectiva. El resto de ítems deben ser 
evaluados de forma cuantitativa de acuerdo a unos rangos de calificación para una mayor reproducibilidad.

Al objetivar los criterios de calificación del profesorado del estudio se propone que los rangos de calificación no sean cerrados de 0-1, sino estratificados. Esta estratificación debería ser consensuada por parte de los centros, los responsables médicos, el tribunal evaluador y la propia Administración.

\section{Agradecimientos}

Agradecimiento al profesorado de Enseñanzas de Régimen Especial, especialidad de danza Clásica, danza Española, danza Contemporánea y Flamenco, como a sus respectivos departamentos en los diferentes Conservatorios Profesionales de Danza y en Escuelas Municipales de Danza deAndalucía. No existen conflictos de interés ni ayudas económicas para la realización del estudio.

\section{Referencias}

Alvero Cruz, J., Marfell Jones, F., Alacid, F. (2014). Comparison of two field methods for estimating body fat in Different spanish dance disciplines. Nutrición Hospitalaria, 30(3), 614-21.

Amada, J. M., González, I., \& Montávez, M. (2013). La expresión corporal: un proyecto para la inclusión Corporal expression: a project for inclusion. Retos. Nuevas Tendencias En Educación Física, Deporte Y Recreación, 24(2004), 107-112. Retrieved from http://www.retos.org/numero_24/monografico24.pdf

Ayala, F., Sainz de Baranda, P., Cejudo, A., \& Santonja, F. (2012). Pruebas angulares de estimacion de la flexibilidad isquiosural: análisis de la fiabilidad y validez. Rev Andal Med Deporte, 5(2), 63-70.

Ayala, F., Sainz de Baranda, P., Cejudo, A., \& Santonja, F. (2013). Pruebas angulares de estimacioin de la flexibilidad isquiosural: descripcioìn de los procedimientos exploratorios y valores de referencia. Rev Andal Med Deporte, 6(3), 120-128.

Ayala, F., Sainz de Baranda, P., De Ste Croix, M., \& Santonja, F. (2012). Reproducibility and Concurrent Validity of Hip Joint Angle Test for Estimating Hamstring Flexibility in Recreationally Active Young Men. Journal of Strength and Conditioning Research. doi:10.1519/ JSC.0b013e31823db1e2

Bautista Arellano, A. (2011). Concepciones de profesores de piano sobre la evaluacioìn. Revista de Educacion, 355, 443-466.

Bejjani, F. J., Halpern, N., Pio, A., Dominguez, R., Voloshin, A., \& Frankel, V. H. (1988). Musculoskeletal demands on flamenco dancers: a clinical and biomechanical study. Foot \& Ankle, 8(5), 254-63. Retrieved from http://www.ncbi.nlm.nih.gov/pubmed/ 3366430

BOJA.(2009a). Corrección de errores de la Orden de 7 de julio de 2009, por la que se regulan las pruebas de aptitud y de acceso a las Enseñanzas Básicas de las Enseñanzas Elementales de Danza en Andalucia. BOJA, 145, 6.

BOJA. (2009b). Decreto 16/2009, de 20 de enero, ha establecido la ordenación y el currículo de las enseñanzas elementales de danza. BOJA, 23, 10-23.

BOJA. (2009c). ORDEN de 24 de junio de 2009, por la que se desarrolla el currículo de las enseñanzas elementales de danza en Andalucía. BOJA, 132, 7-18.

BOJA. (2009d). ORDEN de 7 de julio de 2009, por la que se regulan las pruebas de aptitud y de acceso a las Enseñanzas Básicas de las Enseñanzas Elementales de Danza en Andalucia. BOJA, 145, 1011.

Bozic, P. (2010). Evaluation of the field tests of flexibility of the lower extremity:reability and the concurrent and Factorial Validity. Journal of Strength and Conditioning Research, 24(9), 2523-2531.

Brushøj, C., Langberg, H., Larsen, K., Nielsen, M. B., \& Hölmich, P. (2007). Reliability and normative values of the foot line test: a technique to assess foot posture. The Journal of Orthopaedic and Sports Physical Therapy, 37(11), 703-7. doi:10.2519/ jospt.2007.2525

Carlson, R. E., Fleming, L. L., \& Hutton, W.C.(2000). The biomechanical relationship between the tendoachilles, plantar fascia and metatarsophalangeal joint dorsiflexion angle. Foot \& Ankle International, 21(1), 18-25. Retrieved from http:// www.ncbi.nlm.nih.gov/pubmed/10710257

Clarkson, P. M., Freedson, P. S., Skrinar, M., Keller, B., \& Carney, D. (1989). Anthropometric measurements of adolescent and professional classical ballet dancers. The Journal of Sports Medicine and Physical Fitness, 29(2), 157-62. Retrieved from http:// www.ncbi.nlm.nih.gov/pubmed/2593653

Cole, T. J., Bellizzi, M. C., Flegal, K. M., \& Dietz, W. H. (2000). Establishing a standard definition for child overweight and Obesity Worldwide: International Survey. Bmj, 320, 1-6. doi:10.1136/ bmj.320.7244.1240

Conover, W. (1999). Practical nonparametric statistics (3rd Editio.). Willey: New York.

Dick, F. (2006). The Healthier Dancer Programme. Retrieved from http://www.dansgezond.nl/pdf/jub_artikel_Dick.pdf

Doreste Blanco, J., \& Massó Ortigosa, N. (1989). Perfil fisiológico del bailarín. Archivos De Medicina Del Deporte, 6(21), 57-62.

Drểewska, M., Gauszka, R., \& Sliwiñski, Z. (2012). Hip joint mobility in dancers: preliminary report. Ortopedia, Traumatologia, Rehabilitacja, 14(5), 443-52. doi:10.5604/15093492.1012503

Echegoyen, S.,Aoyama,T., \& Rodríguez, C. (2013). Zapateado technique as an injury risk in Mexican folkloric and Spanish dance: an analysis of execution, ground reaction force, and muscle strength. Medical Problems of Performing Artists, 28(2), 80-3. Retrieved from http:/ /www.ncbi.nlm.nih.gov/pubmed/23752281

Ferrari, E. P., Silva, D.A. S., Martins, C. R., Fidelix, Y. L., \& Petroski, E. L. (2013). Morphological characteristics of professional ballet dancers of the Bolshoi theater company. CollegiumAntropologicum, 37 Suppl 2, 37-43. Retrieved from http://www.ncbi.nlm.nih.gov/ pubmed/23914487

Fetz, F., \& Kornexl, E. (1976). Tests Deportivos motores (Kapelusz.). Argentina.

García, L. S., Medina, F. S., \& Pastor,A. (1996). Exploración clínica del plano sagital del raquis. Selección, 5(2), 88-102.

Garth Fisher, A. (1996). Test y pruebas físicas. Barcelona: Paidotribo.

Gilbert, C. B., Gross, M. T., \& Klug, K. B. (1998). Relationship between hip external rotation and turnout angle for the five classical ballet positions. The Journal of Orthopaedic and Sports Physical Therapy, 27(5), 339-347.

Gomez-Lozano, S., \& Santonja Medina, F. (2012). Columna en Flamenco. Rev Cent Investig Flamenco Telethusa, 5(5), 31-39.

Gras Garcia, E. (1985). Tipificación y baremación de test de Flexibilidad, Equilibrio y Velocidad. De Edwin a Fleishman en una muestra de estudiantes de Educación Física (II). Revista Española de Educación Física Y Deportes., 5, 12-16.

Hergenroeder, A. C., Fiorotto, M. L., \& Klish, W. J. (1991). Body composition in ballet dancers measured by total body electrical conductivity. Medicine and Science in Sports and Exercise, 23(5), 528-33. Retrieved from http://www.ncbi.nlm.nih.gov/pubmed/ 2072830

Husted, J., Cook, R., Farewell, V., \& Gladman, D. (2000). Methods for assessing responsiveness: a critical review and recommendations. $J$ Clin Epidemiol, 53(5), 459-68.

Kerrigan, D. C., Todd, M. K., \& Riley, P. O. (1998). Knee osteoarthritis and high-heeled shoes. Lancet, 351(9113), 1399-401. doi:10.1016/ S0140-6736(97)11281-8

Khan, K. M., Bennell, K., Ng, S., Matthews, B., Roberts, P., Nattrass, C., ... Brown, J. (2000). Can 16-18-year-old elite ballet dancers improve their hip and ankle range of motion over a 12-month period? Clinical Journal of Sport Medicine/: Official Journal of the Canadian Academy of Sport Medicine, 10(2), 98-103. Retrieved from http://www.ncbi.nlm.nih.gov/pubmed/10798790

Liiv, H., Wyon, M. A., Jürimäe, T., Saar, M., Mäestu, J., \& Jürimäe, J. 
(2013). Anthropometry, somatotypes, and aerobic power in ballet, contemporary dance, and dancesport. Medical Problems of Performing Artists, 28(4), 207-11. Retrieved from http:// www.ncbi.nlm.nih.gov/pubmed/24337032

Liiv, H., Wyon, M., \& Jürimae, T. (2014). Anthropometry and somatotypes of competitive DanceSport participants: a comparison of three different styles. Journal of Comparative Human Biology, 65(2), 155-160.

Lopez Gutierrez, C., Linares Girela, D., \& Ruiz Rodriguez, L. (2010). Evaluacioìn de los elementos del proceso de ensenPanza-aprendizaje optimizando la intervencioìn didaìctica en la Educacioìn Fiìsica. Revista Iberoamericana de Educacion, 53 (2), 2-10.

Macintyre, J. (2000). Foot and ankle injuries in dance. Clin Sports Med, 19(2), 351-68.

Marra, M., Caldara, A., Montagnese, C., De Filippo, E., Pasanisi, F., Contaldo, F., \& Scalfi, L. (2009). Bioelectrical impedance phase angle in constitutionally lean females, ballet dancers and patients with anorexia nervosa. European Journal of Clinical Nutrition, 63(7), 905-8. doi:10.1038/ejcn.2008.54

Martinez, E. (2003). La evaluación de la condición física en la educación física. Opinión del profesorado. European Journal of Human Movement, 118-141.

Martínez López, E., Zagalaz Sánchez, M., \& Linares Girela, D. (2003). Las pruebas de aptitud física en la evaluación de la Educación física de la ESO. Apunts: Educacion Fisica Y Deportes, 71, 61-79.

Medina, F. S., Ortuño, R. A., \& Moro, I. M. G. (1994). Angulo lumbo horizontal y valoración de repercusiones del Sindrome de Isquisurales Cortos. Apunts: Educacion Fisica Y Deportes, XXXI, 103-111.

Mihajloviæ, B., \& Mijatov, S. (2003). Body composition analysis in ballet dancers. Medicinski Pregled, 56(11-12), 579-83. Retrieved from http://www.ncbi.nlm.nih.gov/pubmed/15080055

Montaivez, M. (2012). LOE. La consolidacioìn de la Expresioìn Corporal. Esmaìsf. Revista Digital de Educacioìn, 14, 60-80.

Pearson, S. J., \& Whitaker, A. F. (2012). Footwear in classical ballet: a study of pressure distribution and related foot injury in the adolescent dancer. Journal of Dance Medicine \& Science/: Official Publication of the International Association for Dance Medicine \& Science, 16(2), 51-6. Retrieved from http://www.ncbi.nlm.nih.gov/pubmed/ 22687718

Pozo Municio, C. (2003). Perfil antropometrico, biomecanico y clinico del bailarin de Danza Española. Universidad Complutense de Madrid.

Ramos Espada, D., Gonzalez Montesinos, J., \& Mora Vicente, J. (2007). Diferencias en las amplitudes articulares entre varones y mujeres en edad escolar. Apunts. Medicina de l'Esport., 153, 1325.

Ritter, S. (2008). The relationship between lateral ankle sprain and ankle tendinitis in ballet dancers. J Dance Med Sci, 12(1), 23-31.

Russell, J. (2008). Clinical anatomy and biomechanics of the ankle in dance. J Dance Med Sci, 12(3), 75-82.

Russell, J. A., Kruse, D. W., Koutedakis, Y., McEwan, I. M., \& Wyon,
M. A. (2010). Pathoanatomy of posterior ankle impingement in ballet dancers. Clinical Anatomy (New York, N.Y.), 23(6), 613-21. doi:10.1002/ca.20991

Sainz de Baranda, P., \& Santonja, F. (2009). Valoración de la disposición sagital del raquis en gimnastas especialistas en trampolín. Assessment of the sagittal plane of the spine in trampoline gymnasts. International Journal of Sport Science, 5(16), 21-33. doi:10.5232/ ricyde2009.016.02

Santonja Medina, F. (2001). Danza y alteraciones durante el crecimiento. In Librerias Esteban Sanz S.L. (Ed.), Danza y Medicina. Las actas de un encuentro. (pp. 99-108). Librerias Esteban Sanz S.L.

Shrout, P., \& Fleiss, J. (1979). Intraclass correlations: uses in assessing rater reliability. Psychological Bulletin, 86, 420-428.

Sicilia, A. Á., Delgado, M. A., Manzano, J. I., Varela, R., Cañadas, J.F., Gutiérrez, M., ... Movement, H. (2006). La evaluación de aprendizajes en educación física. Diferencias en función del nivel educativo. Motricidad. European Journal of Human Movement., 17, 71-93.

Steinberg, N., Hershkovitz, I., Peleg, S., Dar, G., Masharawi, Y., Zeev, A., \& Siev-Ner, I. (2013). Morphological characteristics of the young scoliotic dancer. Physical Therapy in Sport: : Official Journal of the Association of Chartered Physiotherapists in Sports Medicine, 14(4), 213-20. doi:10.1016/j.ptsp.2012.07.003

Twitchett, E. A., Koutedakis, Y., \& Wyon, M. A. (2009). Physiological fitness and professional classical ballet performance: a brief review. Journal of Strength and Conditioning Research/National Strength \& Conditioning Association, 23(9), 2732-40. doi:10.1519/ JSC.0b013e3181bc1749

Wang, C. L., Hsu, T. C., Shau, Y. W., Shieh, J. Y., \& Hsu, K. H. (1999). Ultrasonographic measurement of the mechanical properties of the sole under the metatarsal heads. Journal of Orthopaedic Research/ : Official Publication of the Orthopaedic Research Society, 17(5), 709-13. doi:10.1002/jor.1100170513

Wilson, M., \& Batson, G (2014). The m/r SEBT: development of a functional screening tool for dance educators. Medical Problems of Performing Artists, 29(4), 207-15. Retrieved from http:// www.ncbi.nlm.nih.gov/pubmed/25433257

Wilson, M., \& Deckert, J. L. (2009). A screening program for dancers administered by dancers. Journal of Dance Medicine \& Science/ : Official Publication of the International Association for Dance Medicine \& Science, 13(3), 67-72. Retrieved from http:// www.ncbi.nlm.nih.gov/pubmed/19754982

Yannakoulia, M., Keramopoulos, A., Tsakalakos, N., \& Matalas, A. L. (2000). Body composition in dancers: the bioelectrical impedance method. Medicine and Science in Sports and Exercise, 32(1), 22834. Retrieved from http://www.ncbi.nlm.nih.gov/pubmed/ 10647554

Yannakoulia, M., Matalas, A. L., Yiannakouris, N., Papoutsakis, C., Passos, M., \& Klimis-Zacas, D. (2004). Disordered eating attitudes: an emerging health problem among Mediterranean adolescents. Eating and Weight Disorders/ : EWD, 9(2), 126-33. Retrieved from http://www.ncbi.nlm.nih.gov/pubmed/15330080
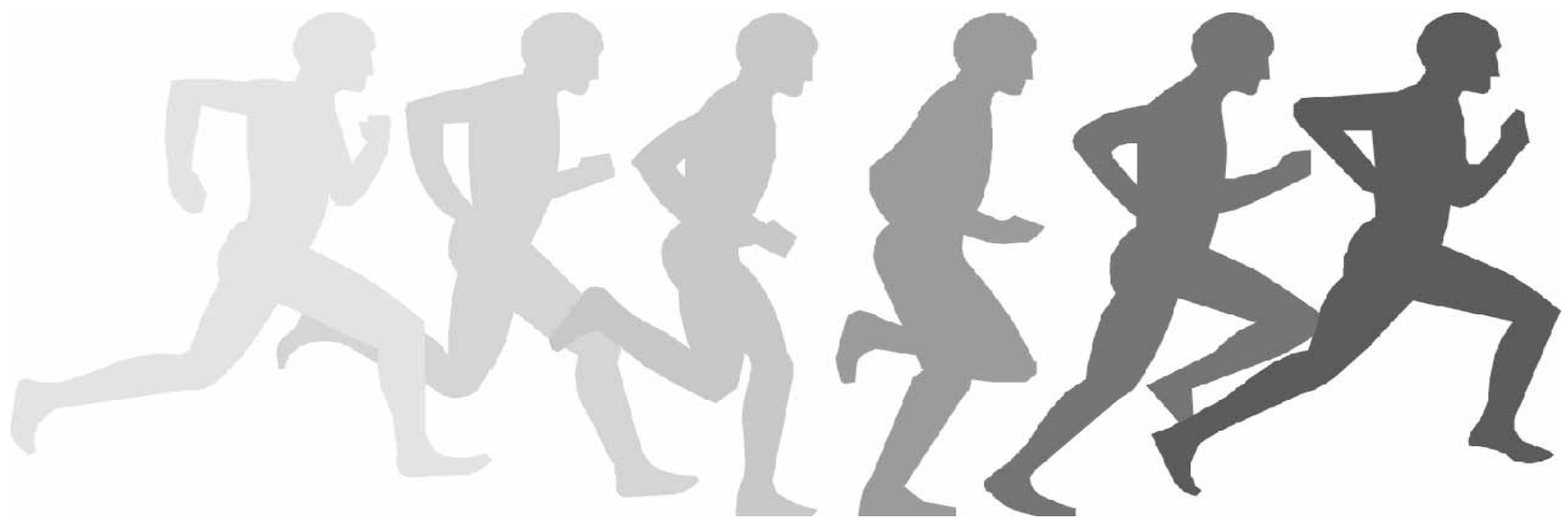